\title{
Características clínicas y mortalidad intrahospitalaria en pacientes con insuficiencia renal aguda en hemodiálisis, en el Hospital Nacional Guillermo Almenara Irigoyen, EsSalud, 2014-2015
}

\author{
Clinical characteristics and in-hospital mortality in patients with acute kidney injury \\ undergoing hemodialysis at the Hospital Nacional Guillermo Almenara Irigoyen, \\ EsSalud, 2014-2015
}

\author{
Antonio Fernandez-Burga ${ }^{1,2, a}$, Kristhian Briceño-Santillán ${ }^{3, b}$, Christian R. Mejia ${ }^{4, c}$ \\ ${ }^{1}$ Hospital Nacional Guillermo Almenara Irigoyen, EsSalud. Lima, Perú. \\ ${ }^{2}$ Facultad de Ciencias de la Salud, Universidad Tecnológica del Perú. Lima, Perú. \\ ${ }^{3}$ Clínica de Hemodiálisis Kidney Care SAC. Lima, Perú. \\ ${ }^{4}$ Coordinación de Investigación, Universidad Continental. Huancayo, Perú. \\ a Médico especialista en nefrología, docente. ORCID: https://orcid.org/0000-0001-9401-1138 \\ ${ }^{\mathrm{b}}$ Médico especialista en nefrologia, ORCID: https://orcid.org/0000-0002-0953-6982 \\ c Doctor en investigación, ORCID: https://orcid.org/0000-0002-5940-7281
}

\section{An Fac med. 2019;80(3):312-6 / DOI: https://10.15381/anales.803.16515}

\begin{abstract}
Correspondencia:
Antonio Manuel Femández Burga amfbxli@gmail.com
\end{abstract}

Recibido: 5 de agosto 2019 Aceptado: 6 de setiembre 2019 Publicación en línea: 30 de setiembre 2019

Conflictos de interés: Los autores declaran no tener conflictos de interés.

Fuente de financiamiento: Autofinanciado

Contribuciones de autoria: AFB tuvo la idea de investigación y recolectó la información, CRM realizó el análisis de los datos. Todos los autores participaron en el análisis, redacción, y dieron su aprobación a la versión final del manuscrito.

Citar como: Femández-Burga A, BriceñoSantillán K, Mejía C. Características clinicas y mortalidad intrahospitalaria en pacientes con insuficiencia renal aguda en hemodiálisis, en el Hospital Nacional Guillermo Almenara Irigoyen, EsSalud, 2014-2015. An Fac med. 2019;80(3):312-6. DOI: https://10.15381/ anales.803.16515

\section{Resumen}

Introducción: No existen datos oficiales sobre los pacientes con insuficiencia renal aguda (IRA) en el Perú. La mayor parte de la información proviene de centros aislados en otros países. Objetivo: Describir las características clínicas y la mortalidad intrahospitalaria en pacientes con insuficiencia renal aguda, en hemodiálisis, atendidos en un hospital de tercer nivel del Perú. Método: Estudio transversal, realizado en pacientes con diagnóstico de IRA en hemodiálisis, atendidos en el Hospital Guillermo Almenara Irigoyen, EsSalud, durante 2014-2015. Se evaluaron las características clínicas generales y se estimó la tasa de mortalidad intrahospitalaria. Resultados: Se evaluaron 183 pacientes. La edad media fue de 58 años y el $57 \%$ fueron varones. El 90\% ingresaron al hospital a través de emergencia (EMG). Los pacientes fueron admitidos en la unidad de cuidados intensivos (UCI), EMG y otras áreas de hospitalización en el $40 \%, 27 \%$ y $33 \%$ de los casos, respectivamente. Las causas más frecuentes de IRA fueron: sepsis (59\%), insuficiencia cardiaca ( $8 \%$ ) y uropatía obstructiva $(7 \%)$. La mortalidad intrahospitalaria fue de $55 \%(n=100)$. Los pacientes fallecidos mostraron una mayor frecuencia de sepsis (71 vs. $38 ; p=0,001$ ) y de compromiso multiorgánico (90 vs. 56; $p<0,001$ ) en comparación con los vivos. Conclusiones: Los pacientes con IRA tuvieron una edad media de 59 años y la mayoría estuvieron hospitalizados en UCI. La causa más frecuente de IRA fue la sepsis. La mortalidad intrahospitalaria fue $55 \%$ y estuvo significativamente asociada a la presencia de sepsis y compromiso multiorgánico.

Palabras clave: Insuficiencia Renal; Diálisis; Mortalidad Hospitalaria; Sepsis (fuente: DeCS BIREME).

\section{Abstract}

Introduction: There are no official data on patients with acute kidney injury (AKI) in Peru. Most of the information comes from isolated centers in other countries. Objective: To describe the clinical characteristics and inhospital mortality in patients with acute kidney injury undergoing hemodialysis at a third level hospital in Peru. Methods: A cross-sectional study. Demographic, clinical, and mortality data were collected from patients diagnosed with AKI undergoing hemodialysis at the Hospital Nacional Guillermo Almenara Irigoyen EsSalud during 2014-2015. Results: 183 patients were included. The average age was 58 years and 57\% were male. Ninety percent of patients were admitted through emergency services (EMG). Patients were hospitalized in the intensive care unit (ICU), EMG rooms and other hospitalization areas in $40 \%, 27 \%$ and $33 \%$ of cases, respectively. The most frequent causes of AKI were sepsis (58\%), heart failure (8\%) and obstructive uropathy. In-hospital mortality rate was $55 \%(n=100)$. The deceased patients showed a higher frequency of sepsis (71 vs. 38; $p=0,001$ ) and multiorgan failure (90 vs. 56; $p<0,001$ ) compared to alive patients. Conclusions: Patients with AKI had a mean age of 59 years and most were hospitalized in the ICU. The most frequent cause of AKI was sepsis. In-hospital mortality was $55 \%$ and was significantly associated with the presence of sepsis and multiorgan failure.

Keywords: Acute Kidney Injury; Dialysis; Hospital Mortality; Sepsis (source: MeSH NLM). 


\section{INTRODUCCIÓN}

La insuficiencia renal aguda (IRA) es un síndrome complejo que puede ocurrir en respuesta a múltiples exposiciones ${ }^{(1)}$ y que generalmente se caracteriza por un abrupto deterioro de la función renal, ocasionando trastornos metabólicos, electrolíticos y en la homeostasis de los fluidos. Su espectro puede variar desde leves incrementos en los parámetros bioquímicos de función renal hasta la falla renal severa tributaria de inicio de terapia de reemplazo renal ${ }^{(2)}$. Según estimaciones, la IRA ocurre en alrededor de 13,3 millones de personas por año, $85 \%$ de las cuales residen en países en vías de desarrollo, relacionándose a 1,7 millones de muertes por año ${ }^{(3)}$.

Algunas de las causas que pueden precipitar un episodio de IRA comprenden los estados hipovolémicos persistentes, la exposición a nefrotóxicos y la sepsis ${ }^{(4)}$. Esta última es considerada la principal causa de muerte en pacientes admitidos en unidades de cuidados intensivos ${ }^{(5)} y$ también se ha reportado a la sepsis como un factor asociado a muerte en pacientes con IRA ${ }^{(6)}$. Otros factores asociados a mortalidad intrahospitalaria son la severidad de la enfermedad al momento del ingreso hospitalario, la magnitud del daño orgánico extra renal, la edad, y la necesidad de diálisis $(6,7,8)$.

La mayor parte de la información publicada sobre este tema proviene de países desarrollados, de modo que, documentar la experiencia local en el manejo de los pacientes con IRA podría contribuir a optimizar los procesos de atención, reducir las complicaciones y mejorar la sobrevida ${ }^{(9)}$. El Hospital Nacional Guillermo Almenara Irigoyen, EsSalud, es un hospital de tercer nivel y uno de los centros de referencia nacional de la seguridad social en el Perú; sin embargo, no posee datos precisos sobre los casos de IRA, sus características y mortalidad asociada.

El objetivo del presente estudio fue describir las características clínicas y la mortalidad intrahospitalaria en pacientes con insuficiencia renal aguda en hemodiálisis atendidos en el Hospital Nacional Guillermo Almenara Irigoyen, EsSalud, en el periodo 2014-2015.

\section{MÉTODOS}

\section{Diseño de estudio}

Se realizó un estudio descriptivo, retrospectivo, y transversal. Se incluyeron los datos de todos los pacientes hospitalizados mayores de 18 años con el diagnóstico de IRA en hemodiálisis atendidos en el Hospital Nacional Guillermo Almenara Irigoyen (HNGAI) del Seguro Social de Salud, EsSalud, entre el 10 de enero de 2014 y el 31 de diciembre de 2015 Se excluyeron aquellos pacientes que experimentaron más de un evento de IRA durante su estancia hospitalaria o que iniciaron hemodiálisis en otro centro sanitario antes de ingresar a nuestro hospital.

\section{Población y muestra}

Se seleccionaron todas las historias clínicas de los pacientes con el diagnóstico de insuficiencia renal aguda en hemodiálisis, obtenidas de la base de datos electrónica de la unidad de hemodiálisis del hospital. Así, no se realizó ningún cálculo de tamaño muestral, y se evaluó a toda la población.

\section{Variables de estudio}

Se identificó el diagnóstico de IRA en hemodiálisis cuando los nefrólogos que evaluaron a los pacientes consignaron esta información en las historias clínicas. El protocolo del servicio de nefrología del HNGAI utiliza los criterios de RIFLE ${ }^{(10)}$ como parámetro para la clasificación de IRA. Se definió como "compromiso multiorgánico" a la presencia de dos o más manifestaciones de compromiso orgánico extrarrenal tales como: shock, insuficiencia respiratoria aguda, insuficiencia hepática aguda, coma y coagulopatía. Estos diagnósticos fueron consignados en las historias clínicas.

Adicionalmente, se revisaron estos registros con la finalidad de obtener los siguientes datos: sexo, edad, forma de ingreso al hospital, servicio de hospitalización, causa de enfermedad, compromiso multiorgánico; así como, la ocurrencia del desenlace muerte durante la hospitalización en la que experimentaron el evento de IRA.

\section{Análisis estadístico}

El análisis estadístico se realizó utilizando el programa STATA Statistical Soft- ware: Versión 14. (College Station, TX: StataCorp LP. $2015^{\circledR}$ ). Se reportaron las frecuencias y porcentajes de las variables cualitativas, así como la media y desviación estándar de las variables cuantitativas. Para el análisis exploratorio se utilizó la prueba chi cuadrado, obteniéndose los valores $p$ de cada cruce, los cuales se consideraron estadísticamente significativos cuándo fueron menores de 0,05.

\section{Aspectos éticos}

Los registros de los pacientes seleccionados fueron identificados por un número arbitrario sin mencionar sus nombres reales, por lo que se preservó la confidencialidad de los datos utilizados. La aplicación del presente estudio se encontró dentro de una investigación con riesgo mínimo debido a que no se realizó ninguna intervención y sólo se recabó información a través del análisis de fuentes secundarias. La investigación fue aprobada por por el Comité de Ética del Hospital Nacional Guillermo Almenara Irigoyen.

\section{RESULTADOS}

Durante el período de estudio se evaluaron 183 pacientes (105 varones y 78 mujeres) con diagnóstico de insuficiencia renal aguda en hemodiálisis. La edad promedio del grupo fue de $59 \pm 18$ años. La principal forma de ingreso al hospital fue a través del servicio de emergencia (90\%) y la mayor parte de los pacientes fueron hospitalizados en la unidad de cuidados intensivos (41\%). Las causas más frecuentes de IRA fueron sepsis, insuficiencia cardíaca aguda y uropatía obstructiva. El $80 \%$ de los pacientes tuvieron un compromiso multiorgánico. El 55\% de los pacientes fallecieron durante su hospitalización (Tabla 1).

El análisis comparativo de las características clínicas mostró diferencias estadísticamente significativas entre los grupos de pacientes fallecidos y vivos, respecto a la causa de IRA y la existencia de compromiso multiorgánico (Tabla 2). Los pacientes fallecidos mostraron una mayor frecuencia de sepsis (71 vs. 38; $p$ =0,001) y de compromiso multiorgánico (90 vs. 56 ; $p<0,001$ ) y una menor fre- 
Tabla 1. Características clínicas de los pacientes con insuficiencia renal aguda en hemodiálisis, en el Hospital Nacional Guillermo Almenara Irigoyen, EsSalud, 2014-2015. $(n=183)$.

\begin{tabular}{|c|c|c|}
\hline Características & $\mathbf{n}$ & $\%$ \\
\hline \multicolumn{3}{|l|}{ Edad } \\
\hline 18-64 años & 106 & 58 \\
\hline$>64$ años & 77 & 42 \\
\hline \multicolumn{3}{|l|}{ Sexo } \\
\hline Femenino & 78 & 43 \\
\hline Masculino & 105 & 57 \\
\hline \multicolumn{3}{|l|}{ Forma de ingreso } \\
\hline Emergencia & 165 & 90 \\
\hline Consultorio externo & 18 & 10 \\
\hline \multicolumn{3}{|l|}{ Servicio de hospitalización } \\
\hline Unidad de cuidados intensivos & 75 & 41 \\
\hline Salas de emergencia & 49 & 27 \\
\hline Hospitalización general & 59 & 32 \\
\hline \multicolumn{3}{|l|}{ Causa de insuficiencia renal aguda } \\
\hline Sepsis & 109 & 59 \\
\hline Insuficiencia cardíaca aguda & 15 & 8 \\
\hline Uropatía obstructiva & 12 & 7 \\
\hline Hipovolemia & 11 & 6 \\
\hline Enfermedades autoinmunes & 7 & 4 \\
\hline Otros & 29 & 16 \\
\hline \multicolumn{3}{|l|}{ Compromiso multiorgánico } \\
\hline Sí & 146 & 80 \\
\hline No & 37 & 20 \\
\hline \multicolumn{3}{|l|}{ Fallece } \\
\hline Sí & 100 & 55 \\
\hline No & 83 & 45 \\
\hline
\end{tabular}

cuencia de insuficiencia cardiaca aguda (4 vs. $11 ; p=0,023$ ) y uropatía obstructiva (2 vs. $10 ; p=0,006)$, en comparación con los pacientes vivos.

\section{DISCUSIÓN}

La presente investigación constituye un análisis inicial de una de las series más grandes de pacientes con insuficiencia renal aguda estudiadas en el Perú, en donde el $55 \%$ de pacientes fallecieron durante su hospitalización, existiendo asociación entre este desenlace y la presencia de sepsis y compromiso multiorgánico.

La mortalidad asociada a un evento de insuficiencia renal aguda varía de acuerdo con su severidad, siendo mayor en los pacientes que requieren terapia dialítica ${ }^{(11)}$. Esto puede ser explicado por el mayor compromiso sistémico así como por la elevada frecuencia de falla multiorgánica observada en este

Tabla 2. Comparación de las características según mortalidad, de los pacientes con insuficiencia renal aguda, en el Hospital Nacional Guillermo Almenara Irigoyen, EsSalud, 2014-2015.

\begin{tabular}{|c|c|c|c|}
\hline Variable & $\begin{array}{l}\text { Vivos } \\
\text { n (\%) }\end{array}$ & $\begin{array}{c}\text { Fallecidos } \\
\text { n (\%) }\end{array}$ & Valor p ${ }^{+}$ \\
\hline \multicolumn{4}{|l|}{ Edad } \\
\hline $18-64$ & $49(46,2)$ & $57(53,8)$ & \multirow{2}{*}{0,781} \\
\hline$>64$ & $34(44,2)$ & $43(55,8)$ & \\
\hline \multicolumn{4}{|l|}{ Sexo } \\
\hline Masculino & $42(40,0)$ & $63(60,0)$ & \multirow{2}{*}{0,091} \\
\hline Femenino & $41(52,6)$ & $37(47,4)$ & \\
\hline \multicolumn{4}{|l|}{ Forma de ingreso } \\
\hline EMG & $73(44,2)$ & $92(55,8)$ & \multirow{2}{*}{0,360} \\
\hline CCEE & $10(55,6)$ & $8(44,4)$ & \\
\hline \multicolumn{4}{|l|}{ Servicio de hospitalización } \\
\hline $\mathrm{UCl}$ & $30(40,0)$ & $45(60,0)$ & \multirow{3}{*}{0,070} \\
\hline Emergencia & $19(38,8)$ & $30(61,2)$ & \\
\hline Hospitalización General & $34(57,6)$ & $25(42,4)$ & \\
\hline \multicolumn{4}{|c|}{ Causa de insuficiencia renal aguda } \\
\hline \multicolumn{4}{|l|}{ Sepsis } \\
\hline $\mathrm{Si}$ & $38(34,9)$ & $71(65,1)$ & \multirow{2}{*}{0,001} \\
\hline No & $45(60,8)$ & $29(39,2)$ & \\
\hline \multicolumn{4}{|l|}{ Insuficiencia cardiaca } \\
\hline $\mathrm{Si}$ & $11(73,3)$ & $4(26,7)$ & \multirow{2}{*}{0,023} \\
\hline No & $72(42,9)$ & $96(57,1)$ & \\
\hline \multicolumn{4}{|l|}{ Uropatía Obstructiva } \\
\hline $\mathrm{Si}$ & $10(83,3)$ & $2(16,7)$ & \multirow{2}{*}{0,006} \\
\hline No & $73(42,7)$ & $98(57,3)$ & \\
\hline \multicolumn{4}{|l|}{ Hipovolemia } \\
\hline $\mathrm{Si}$ & $5(45,5)$ & $6(54,5)$ & \multirow{2}{*}{0,995} \\
\hline No & $78(45,4)$ & $94(54,6)$ & \\
\hline \multicolumn{4}{|c|}{ Enfermedades Autoinmunes } \\
\hline $\mathrm{Si}$ & $4(57,1)$ & $3(42,9)$ & \multirow{2}{*}{0,523} \\
\hline No & $79(44,9)$ & $97(55,1)$ & \\
\hline \multicolumn{4}{|c|}{ Compromiso Multiorganico } \\
\hline $\mathrm{Si}$ & $56(38,4)$ & $90(61,4)$ & \multirow{2}{*}{$<0,001$} \\
\hline No & $27(73,0)$ & $10(27,0)$ & \\
\hline
\end{tabular}

'Prueba de Chi cuadrado.

EMG: Emergencia, CCEE: Consultorios externos, UCI: Unidad de cuidados intensivos

grupo de pacientes ${ }^{(12)}$, lo cual guarda concordancia con lo hallado en nuestra investigación. Así, Riffaut y colaboradores documentaron una mortalidad intrahospitalaria de $51 \%$ en pacientes con insuficiencia renal aguda que recibieron terapia de reemplazo renal ${ }^{(13)}$; mientras que Lu y colaboradores reportaron una mortalidad intrahospitalaria de $44 \%$ en pacientes con insuficiencia renal aguda severa ${ }^{(12)}$. En el Perú, se han reporta- do tasas de mortalidad intrahospitalaria en pacientes con insuficiencia renal aguda cercanas al $40 \%{ }^{(6,14,15)}$; sin embargo, los criterios de inclusión utilizados en estos estudios fueron diferentes y el número de participantes fue menor respecto a nuestra investigación.

La evidencia actual reporta que los pacientes que experimentan un evento de insuficiencia renal aguda residentes 
de países en vías de desarrollo, como el Perú, son jóvenes, tienen una etiología unifactorial y pocas comorbilidades (3), lo cual contrasta con nuestros hallazgos que muestran que los pacientes tenían una mayor edad, diversas etiologías e importantes comorbilidades. Este escenario, aparentemente contradictorio, es explicado por el perfil de los pacientes admitidos en hospitales generales de referencia (como el HNGAI), los cuales comparten características con los pacientes atendidos en países desarrollados (3). Resultados similares a los nuestros fueron observados en dos estudios realizados en hospitales públicos del Perú, según los cuales los pacientes con insuficiencia renal aguda eran mayores de 50 años, provenían mayoritariamente de la unidad de cuidados intensivos ${ }^{(14,15)}$ y padecían de múltiples complicaciones extrarrenales ${ }^{(14)}$.

Un hallazgo estadísticamente significativo de nuestro trabajo fue la mayor frecuencia de sepsis en los pacientes fallecidos en comparación con los vivos. Al respecto, la sepsis es considerada la causa más frecuente de insuficiencia renal aguda en pacientes críticamente enfermos ${ }^{(6,16,17,18)}$. Además, existe evidencia de que la insuficiencia renal aguda secundaria a sepsis se asocia a una mayor mortalidad en comparación con la insuficiencia renal aguda no séptica ${ }^{(19,20,21)}$. Un estudio realizado en nuestro país reportó una prevalencia de sepsis de $64 \%$ en pacientes hospitalizados con insuficiencia renal aguda ${ }^{(22)}$, mientras que un estudio realizado en Brasil reportó una prevalencia de sepsis de $52 \%$ en pacientes con insuficiencia renal aguda que requirieron diálisis, siendo considerada un factor de riesgo independiente para mortalidad ${ }^{(23)}$. Otro estudio en Perú, también demostró asociación significativa entre la presencia de sepsis y la sobrevida de pacientes con insuficiencia renal aguda ${ }^{(6)}$. Estos resultados desfavorables estarían relacionados a ciertas alteraciones fisiopatológicas particulares de la sepsis como hiperemia, vasodilatación, y apoptosis tubular renal aguda que participarían en la generación de un mayor daño ${ }^{(24)}$.

También observamos una elevada frecuencia de compromiso multiorgánico
(80\%), la cual fue significativamente mayor en los pacientes fallecidos en comparación con los vivos. Un estudio realizado en el ámbito local observó resultados similares al demostrar asociación entre la presencia de shock, disfunción respiratoria, disfunción cardíaca y mortalidad ${ }^{(6)}$. Experiencias en otras realidades han reportado asociación entre mortalidad y necesidad de ventilación mecánica ${ }^{(23)}$, así como con la magnitud del fallo orgánico extrarrenal ${ }^{(11)}$ en pacientes con insuficiencia renal aguda. Es importante señalar que el $66 \%$ de los pacientes que presentaron compromiso multiorgánico en nuestra muestra, también tuvieron sepsis. Al respecto, se ha descrito un mayor compromiso multisistémico en los pacientes con sepsis ${ }^{(19)}$, producto de un intenso estado inflamatorio ${ }^{(25)}$, el cual se traduce en una mayor necesidad de soporte vital con uso de ventilación mecánica, drogas vasoactivas y fluidos ${ }^{(19,26,27)}$.

Es importante reconocer algunas limitaciones de nuestro trabajo. El diagnóstico de insuficiencia renal aguda fue tomado de las historias clínicas, por tanto, estuvo sujeto a heterogeneidad por parte de los médicos que evaluaron a los pacientes. Además, no estuvieron disponibles otras mediciones como resultados laboratoriales o scores de severidad que hubiesen permitido caracterizar mejor a la población de estudio. Asimismo, la investigación se realizó en un solo centro; sin embargo, representa la primera comunicación de mortalidad asociada a IRA en pacientes de la seguridad social del Perú. Consideramos que nuestros hallazgos representan una mirada inicial a una realidad muy poco estudiada en el país, y plantea importantes hallazgos que podrían servir como hipótesis para futuras investigaciones.

Concluimos que en el periodo de estudio, los pacientes con IRA en hemodiálisis en el HNGAl tuvieron una edad media de 59 años y la mayor parte estuvieron hospitalizados en la unidad de cuidados intensivos. La causa más frecuente de IRA fue la sepsis. La mortalidad intrahospitalaria fue de $55 \%$ y estuvo asociada a la presencia de sepsis y compromiso multiorgánico.

\section{AGRADECIMIENTOS}

Al Dr. Wilfredo Cortez Sanchez, Jefe del Servicio de Nefrología del Hospital Nacional Guillermo Almenara Irigoyen y a todo el personal asistencial y administrativo que labora en el servicio. A la Mg. Kelly Achachao Almerco por su colaboración en la revisión metodológica.

\section{REFERENCIAS BIBLIOGRÁFICAS}

1. Endre Z, Kellum J, Di Somma S, Doi K, Goldstein S, Koyner J, Macedo E. Differential Diagnosis of AKI in Clinical Practice by Functional and Damage Biomarkers. Contrib Nephrol. 2013;182:30-44. DOI: 10.1159/000349964

2. Rewa O, Bagshaw SM. Acute kidney injury: epidemiology, outcomes and economics. Nat Rev Nephrol. 2014;10(4):193-207. DOI: 10.1038/ nrneph.2013.282

3. Mehta RL, Cerdá J, Burdamann EA, Tonelli M, Garcia-Garcia G, Jha V, et al. International Society of Nephrology's 0by25 initiative for acute kidney injury. Lancet. 2015;385(9987):2616-43. DOI: 10.1016/S0140-6736(15)60126-X

4. Bellomo R, Kellum JA, Ronco C. Acute kidney injury. Lancet. 2012;380:756-66. DOI: 10.1016/ S0140-6736(11)61454-2

5. Angus DC, van der Poll T. Severe sepsis and septic shock. N Engl J Med. 2013;369:840-851. DOI: 10.1056/NEJMra1208623

6. León C, Cieza J, Valenzuela R. Injuria renal aguda, perfil epidemiológico intrahospitalario y factores asociados al riesgo de muerte. Rev Medica Hered. 2014;25:189-195.

7. Clec'h C, Gonzalez F, Lautrette A, Nguile-Makao M, Garrouste-Orgeas M, Jamali S. Multiple-center evaluation of mortality associated with acute kidney injury in critically ill patients: a competing risks analysis. Crit Care. 2011;15(3): R128. DOI:10.1186/ cc10241

8. Bucuvic E, Ponce D, Balbi A. Risk factors for mortality in acute kidney injury. Rev Assoc Med Bras. 2011;57(2):156-161. DOI: 10.1590/S010442302011000200012

9. Susantitaphong P, Cruz D, Cerda J, Abulfaraj M, Alqahtani F, Koulouridis I, et al. World incidence of AKI: a meta-analysis. Clin J Am Soc Nephrol. 2013;8(9):1482-93. DOI: 10.2215/CJN.00710113

10. Bellomo R, Ronco C, Kellum JA, Mehta RL, Palevsky P; Acute Dialysis Quality Initiative workgroup. Acute renal failure - definition, outcome measures, animal models, fluid therapy and information technology needs: the Second International Consensus Conference of the Acute Dialysis Quality Initiative (ADQI) Group. Crit Care. 2004;8:R204-R212. DOI: 10.1186/cc2872

11. Palevsky PM, Zhang JH, O'Connor TZ, et al. Intensity of renal support in critically ill patients with acute kidney injury. N Engl J Med 2008;359:7-20. DOI: 10.1056/NEJMoa0802639

12. Lu R, Muciño-Bermejo M, Armignacco P, Fang $Y$, Cai H, Zhang M, et al. Survey of acute kidney injury and related risk factors of mortality in hospitalized patients in a third-level urban hospital of Shanghai. Blood Purif. 2014; 38:140-148. DOI: 10.1159/000366127 
13. Riffaut N, Moranne O, Hertig A, Hannedouche T, Couchoud C. Outcomes of acute kidney injury depend on initial clinical features: a national French cohort study. Nephrol Dial Transplant. 2018;33: 2218-2227. DOI: 10.1093/ndt/gfy137

14. Herrera-Añazco $P$, Taype-Rondan A, PachecoMendoza J, Miranda JJ. Factors associated with mortality in a population with acute kidney injury undergoing hemodialysis in Peru. J Bras Nefrol. 2017; 39(2):119-125. DOI: 10.5935/0101-2800.20170029

15. Herrera-Añazco P, Palacios-Guillén M, ChipayoGonzales D, Gavidia-Calderón J, Silveira-Chau M. Características clínicas de los pacientes con injuria renal aguda en diálisis en un hospital general. An Fac med. 2013;74(4):307-9.

16. Yokota L, Sampaio B, Rocha E, Balbi A, Ponce D: Acute kidney injury in elderly intensive care patients from a developing country: clinical features and outcome. Int J Nephrol Renovasc Dis. 2017;10: 27-33. DOI: 10.2147/IJNRD.S126534

17. Kellum J, Prowle J: Paradigms of acute kidney injury in the intensive care setting. Nat Rev Nephrol. 2018 Apr;14(4):217-230. DOI: 10.2147/ IJNRD.S126534
18. Godin M, Murray P, Mehta R. Clinical Approach to the Patient with $A K I$ and Sepsis. Semin Nephrol. 2015; 35(1): 12-22. DOI: 10.1016/j.semnephrol.2015.01.003.

19. Bagshaw SM, Uchino S, Bellomo R, Morimatsu H, Morgera S, Schetz M, et al. Septic acute kidney injury in critically ill patients: clinical characteristics and outcomes. Clin J Am Soc Nephrol. 2007;2(3):431-9.

20. Oppert M, Engel C, Brunkhorst FM, Bogatsch $\mathrm{H}$, Reinhart K, Frei $\mathrm{U}$, et al. Acute renal failure in patients with severe sepsis and septic shock a significant independent risk factor for mortality: results from the German Prevalence Study. Nephrol Dial Transplant. 2008, 23:904-9.

21. Yegenaga I, Hoste E, Van Biesen W, Vanholder R, Benoit D, Kantarci G, et al. Clinical characteristics of patients developing ARF due to sepsis/systemic inflammatory response syndrome: results of a prospective study. Am J Kidney Dis. 2004;43:817-824.

22. Lluncor J, Cruz-Encarnación M, Cieza J. Factores asociados a injuria renal aguda en pacientes incidentes de un hospital general de Lima-Perú. Rev Med Hered. 2015;26:24-30.
23. Peres LA, Wandeur V, Matsuo T. Predictors of acute kidney injury and mortality in an Intensive Care Unit. J Bras Nefrol 2015;37:38-46. DOI: 10.5935/01012800.20150007.

24. Wan L, Bagshaw SM, Langenberg C, Saotome T, May C, Bellomo R.: Pathophysiology of septic acute kidney injury: What do we really know? Crit Care Med. 2008;36 (4 Suppl): S198-S203. DOI: 10.1097/ CCM.0b013e318168ccd5

25. Alobaidi R, Basu R, Goldstein S, Bagshaw S Sepsis-Associated Acute Kidney Injury. Semin Nephrol. 2015; 35(1):2-11. DOI: 10.1016/j.semnephrol.2015.01.002

26. Martin CM, Priestap F, Fisher H, et al; STAR Registry Investigators. A prospective, observational registry of patients with severe sepsis: the Canadian Sepsis Treatment and Response Registry. Crit Care Med. 2009; 37(1):81-8. DOI: 10.1097/ CCM.0b013e31819285f0

27. Hoste EA, Lameire NH, Vanholder RC, Benoit DD Decruyenaere JM, Colardyn FA. Acute renal failure in patients with sepsis in a surgical ICU: predictive factors, incidence, comorbidity, and outcome. J Am Soc Nephrol. 2003;14:1022-30. 